\title{
Effet combiné de l'oxygène et de la température sur l'acidification et les productions de diacétyle et d'acétoïne par Lactococcus lactis subsp lactis biovar diacetylactis
}

\author{
N Bassit, E Latrille, CY Boquien *, D Picque, G Corrieu \\ Laboratoire de génie des procédés biotechnologiques agro-alimentaires, INRA, \\ 78850 Thiverval-Grignon, France
}

(Reçu le 28 juillet 1993; accepté le 18 janvier 1994)

\begin{abstract}
Résumé - L'effet de la concentration initiale en oxygène dissous $(100,50,21$ et $0 \%$ de la saturation du milieu par l'oxygène) et de la température de culture $\left(30,26,22\right.$ et $\left.18^{\circ} \mathrm{C}\right)$ sur la vitesse d'acidification et sur les productions de diacétyle et d'acétoïne résultant de la croissance de Lactococcus lactis subsp lactis biovar diacetylactis a été étudié. Les résultats sont présentés sous forme de surface de réponse. Les effets linéaires, quadratiques et d'interaction de la température et de la concentration en oxygène ont été étudiés après une reparamétrisation en fonction des polynômes orthogonaux. L'effet de la température est plus marqué que celui de l'oxygène, dans le seul cas de l'acidification. Un effet inverse est obsenvé pour la production de diacétyle et d'acétoïne et pour le rapport des concentrations de ces 2 produits (D/A). Des optimums locaux ont été trouvés aux limites du plan d'expérience utilisé. Ainsi la concentration en diacétyle et le rapport D/A présentent un maximum à $18^{\circ} \mathrm{C}$ et $100 \%$ d'oxygène, égal respectivement à $0,31 \mathrm{mmol} / \mathrm{l}$ et $5,9 \%$. La concentration en acétoïne atteint son maximum $(5,3$ $\mathrm{mmol} / \mathrm{l})$ à $26^{\circ} \mathrm{C}$ et à $100 \%$ d'oxygène. En revanche, c'est à $30^{\circ} \mathrm{C}$ et en absence d'oxygène que la vitesse maximale d'acidification est la plus élevée $(0,23$ unité $\mathrm{pH} / \mathrm{h})$. Un effet d'interaction de la température et de la concentration initiale en oxygène a été également mis en évidence pour les 4 variables étudiées.
\end{abstract}

diacétyle / acetoïne / acidification / Lactococcus lactis subsp lactis

Summary - Combined effect of oxygen and temperature on acidification and production of diacetyl and acetoin by Lactococcus lactis subsp lactis biovar diacetylactis. The effect of initial oxygen concentration $(100,50,21$ and $0 \%$ of the medium saturation by oxygen) and temperature $(30,26$, 22 and $18^{\circ} \mathrm{C}$ ) on the rate of acidification and on diacetyl and acetoin production by Lactococcus lactis

\footnotetext{
* Correspondance et tirés à part
} 
subsp lactis biovar diacetylactis was studied. The results were described by the methodology of response surface. Linear, squared and simple interaction effects were estimated after an orthogonal polynomial reparametrisation. The effect of temperature was higher than the effect of oxygen for acidification only. The opposite effect was observed for diacetyl and acetoin production and consequently for the ratio diacetylacetoin (D/A). Optima were found on the limits of the experimental design. Diacetyl concentration and D/A ratio reached a maximum value at $18^{\circ} \mathrm{C}$ and $100 \%$ of oxygen concentration ( $0.3 \mathrm{mmol} / \mathrm{l}$ and $5.9 \%$, respectively). Acetoin concentration was maximum ( $5.3 \mathrm{mmol} / \mathrm{l})$ at $26^{\circ} \mathrm{C}$ and $100 \%$ of oxygen concentration. On the other hand, the maximum acidification rate reached its maximum value ( 0.23 unit $\mathrm{pH} / \mathrm{h})$ at $30^{\circ} \mathrm{C}$ and $0 \%$ of oxygen concentration. For the four variables tested, there was a significant interaction between temperature and initial oxygen concentration.

diacetyl / acetoin / acidification / Lactococcus lactis subsp lactis

\section{INTRODUCTION}

Les souches de Lactococcus lactis subsp lactis biovar diacetylactis se distinguent des autres bactéries lactiques par leur aptitude à utiliser le citrate pour produire du diacétyle et de l'acétoïne. Ces composés contribuent de façon prépondérante à la qualité organoleptique des fromages frais. Leur production dépend de plusieurs facteurs : la souche utilisée, la composition du milieu de culture (Kaneko et al, 1990), les conditions de fermentation ( $\mathrm{pH}$, temperature, aération), le mode de culture (culture en cellules libres ou immobilisées) (Ochi et al, 1991). Des travaux récents ont montré que la présence d'oxygène favorisait la synthèse de diacétyle et d'acétoïne et limitait l'acidification (Takahashi et al, 1990 ; Bassit et al, 1993). Bassit et al (1994) ont également montré qu'une température élevée favorisait l'acidification et réduisait la production des composés d'arôme, notamment le diacétyle. Cela confirme les résultats de Petit et al (1989) selon lesquels l'accumulation du diacétyle dans le milieu est d'autant plus importante que la température de fermentation est basse.

Dans cet article, nous décrivons l'effet combiné de la concentration initiale en oxygène dissous et de la température de culture sur la vitesse d'acidification et sur les concentrations maximales de diacétyle et d'acétoïne produites par Lactococcus lactis subsp lactis biovar diacetylactis.

\section{MATÉRIELS ET MÉTHODES}

\section{Micro-organisme}

La souche utilisée est $L$ lactis subsp lactis biovar diacetylactis CNRZ 483. Elle provient de la collection de bactéries lactiques de l'INRA de Jouy-en-Josas (France). Elle est stockée dans du lait tournesolé à $-20^{\circ} \mathrm{C}$.

\section{Milieu de culture et conditions de fermentation}

Le milieu de culture est composé de lait écrémé en poudre ("Elle et Vire" ULN, Condé s/Vire, France) reconstitué à $10 \%(\mathrm{v} / \mathrm{v})$ d'extrait sec et pasteurisé à $92^{\circ} \mathrm{C}$ pendant $5 \mathrm{~min}$. Il est additionné d'extrait de présure à $0,1 \%$ (Boll, Arpajon, France).

Quarante $\mathrm{ml}$ de lait ensemencé et emprésuré sont déposés dans des Erlen-Meyer stériles, d'un volume de $150 \mathrm{ml}$. Le milieu de culture est saturé, soit par de l'oxygène, soit par un mélange d'oxygène et d'air ( $50 \%$ d'oxygène), soit par de l'air ( $21 \%$ d'oxygène), soit par de l'azote. Après $3 \mathrm{~min}$ de balayage par le gaz choisi, les Erlen sont fermés hermétiquement et incubés à la température choisie sans agitation. Les prélèvements sont effectués toutes les 1 à $2 \mathrm{~h}$ selon la température de culture. Chaque Erlen correspond à un prélèvement sur lequel différentes analyses sont effectuées. 


\section{Méthodes d'analyse biochimique}

Les concentrations en acétoïne sont déterminées, sur les prélèvements, par chromatographie liquide haute performance (Xanthopoulos et al, 1994). Le diacétyle est quantifié par méthode colorimétrique (Walsh et Cogan, 1974). En moyenne, pour une expérience, 10 échantillons sont analysés.

\section{Définition des 4 variables étudiées}

Les variables étudiées sont la vitesse maximale d'acidification $\left(\mathrm{V}_{\mathrm{pH}}\right)$, la concentration maximale de diacétyle $\left(C_{D}\right)$, la concentration maximale d'acétoïne $\left(\mathrm{C}_{A}\right)$ et le rapport de ces concentrations (D/A en $\left.\%=100^{\circ} \mathrm{C}_{D} / \mathrm{C}_{A}\right)$. Ce rapport permet de caractériser la qualité du caillé qui est jugée d'autant meilleure que ce rapport est élevé.

Les mesures du $\mathrm{pH}$, effectuées pendant les cultures, ont été ajustées par la fonction de Weibull (Lebreton et Millier, 1982) qui s'écrit :

$$
p H(t)=p H_{0}-a(1-\exp (-b(q))
$$

où $\mathrm{a}, \mathrm{b}, \mathrm{c}$ sont des constantes, t est le temps (h), $\mathrm{pH}_{0}$ la valeur du $\mathrm{pH}$ au temps initial $(\mathrm{t}=0)$. La vitesse maximale d'acidification $\mathrm{V}_{\mathrm{pH}}$ exprimée en unité $\mathrm{pH} / \mathrm{h}$ est la valeur pour laquelle la dérivée seconde de cette fonction s'annule (Spinnler et Corrieu, 1989).

\section{Plan d'expérience et analyse des effets des facteurs}

Pour des raisons de simplicité expérimentale, un plan d'expérience complet à 2 facteurs (température et concentration initiale en oxygène) et à 4 niveaux $(100,50,21,0 \%$ d'oxygène et 30,26 , $22,18^{\circ} \mathrm{C}$ ) a été réalisé. Afin d'assurer une bonne reproductibilité des phénomènes, chaque expérence a été effectuée 2 fois, soit un total de 32 expériences. L'existence d'essais effectués à $21 \%$ d'oxygène (correspondant à la composition de l'air) dans la planification expérimentale entraîne une répartition non équidistante des niveaux de concentration en oxygène. L'analyse des effets a donc été conduite après une reparamétrisation en fonction des polynômes orthogonaux (Kobilinsky, 1988). En supposant un modèle de réponse du second ordre pour cha- cune des 4 variables expliquées $\mathrm{V}_{\mathrm{pH}}, \mathrm{C}_{\mathrm{D}}, \mathrm{C}_{\mathrm{A}}$ et $D / A$, nous avons comparé entre eux les effets linéaires, quadratiques et d'interaction simple de la température et de la concentration initiale en oxygène. La répétition des expérimentations a permis de calculer le manque d'ajustement de chaque modèle afin de valider les hypothèses de modélisation.

La construction des polynômes orthonormés et la détermination des effets ont été réalisées à l'aide du logiciel statistique Splus (version 2.3 UNIX) en utilisant la procédure Isfit sur le modèle reparamétrisé.

Les modèles établis ont permis une représentation sous forme de surface de réponse pour les 4 variables à laide des procédures urégression de surface de réponsen (RSREG) et G3D du logiciel SAS (Statistic Analytic System Version 6.07 UNIX).

La variable expliquée $(Y)$ peut être exprimée en fonction des 2 variables explicatives que sont la température $(T)$ et la concentration initiale en oxygène $\left(\mathrm{O}_{2}\right)$ et d'une erreur de mesure $\varepsilon$. Le modèle s'écrit alors sous la forme :

$$
\begin{aligned}
Y= & a_{00}+a_{10} \cdot T+a_{01} \cdot O_{2}+a_{20} \cdot T^{2} \\
& +a_{02} \cdot O_{2}^{2}+a_{11} \cdot T \cdot O_{2}+\varepsilon
\end{aligned}
$$

Les coefficients $a_{i j}$ intervenant dans cette forme du modèle ne sont pas interprétables. Pour obtenir des coefficients interprétables, on réécrit (1) en fonction des polynômes orthonormés de $\mathrm{T}_{\text {et }} \mathrm{O}_{2}$ dont la construction est donnée en annexe (Noël et al, 1991). Dans l'expression obtenue :

$$
\begin{gathered}
Y\left(T, O_{2}\right)=\alpha_{00}+\alpha_{10} P_{1}(T)+\alpha_{01} Q_{1}\left(O_{2}\right) \\
+\alpha_{20} P_{2}(T)+\alpha_{02} Q_{2}\left(O_{2}\right)+\alpha_{11} P_{1}(T) Q_{1}\left(O_{2}\right)+\varepsilon
\end{gathered}
$$

les coefficients s'interprètent comme suit :

$-\alpha_{00}$ est l'effet moyen (moy);

$-\alpha_{10}$ est l'effet linéaire de la température (linT);

$-\alpha_{01}$ est l'effet linéaire de la concentration en $\mathrm{O}_{2}$ $\left(\right.$ lin $\left.\mathrm{O}_{2}\right)$;

$-\alpha_{20}$ est l'effet quadratique de la température (quadT);

$-\alpha_{02}$ est l'effet quadratique de la concentration en $\mathrm{O}_{2}$ (quad $\mathrm{O}_{2}$ );

$-\alpha_{11}$ est l'effet d'interaction simple de la température et de la concentration en $\mathrm{O}_{2}\left(\right.$ linT.lin $\left.\mathrm{O}_{2}\right)$.

Le fait de normer les polynômes orthogonaux rend les effets comparables. En explicitant les polynômes $P_{i}$ et $Q_{i}$, on obtient l'équation suivante : 


$$
\begin{gathered}
Y\left(T, O_{2}\right)=\alpha_{00}+\alpha_{10}(0,224 \cdot T-5,366) \\
+\alpha_{01}\left(0,0266 \cdot O_{2}-1,1394\right)+\alpha_{20}(0,0625 \\
\left.\cdot T^{2}-3 \cdot T+34,75\right)+\alpha_{02}\left(0,00093 \cdot O_{2}^{2}\right. \\
\left.-0,096 \cdot O_{2}+1,0943\right)+\alpha_{11}(0,224 \cdot T-5,366) \\
\left(0,0266 \cdot O_{2}-1,1394\right)+\varepsilon
\end{gathered}
$$

T est exprimée en ${ }^{\circ} \mathrm{C}$ et $\mathrm{O}_{2}$ en pourcentage de la saturation du milieu par l'oxygène.

\section{RÉSULTATS}

\section{Ajustement du modèle et analyse des effets}

Les tableaux I à IV donnent pour chacune des variables $V_{p H}, C_{D}, C_{A}$ et D/A les estimations $\hat{\alpha}_{i j}$ des effets linéaires, quadratiques et d'interaction simple de la température et de la concentration en oxygène, les écart types de ces estimations, les $t$ de Student et probabilités associées. Ils donnent aussi le coefficient de détermination $R^{2}$, le $F$ de Fisher permettant de tester le défaut d'ajustement du modèle et la probabilité associée.

Les tests $\mathrm{F}$ de défaut d'ajustement sont non significatifs pour chacune des 4 variables, ce qui montre que l'approximation quadratique est dans tous les cas suffisante pour décrire les données.

On constate que la température et l'oxygène ont dans tous les cas un effet de type linéaire et/ou quadratique significatif ; l'interaction est également toujours significative.

\begin{tabular}{|c|c|c|c|c|}
\hline$V_{p H}$ & coef & std.err & t.stat & $P(t)$ \\
\hline $\begin{array}{l}\text { Moy* } \\
\text { linT. } \\
\text { linO }{ }_{2}^{*} \\
\text { quadT } \\
\text { quadO } \\
\text { linT.linO }_{2}{ }^{*}\end{array}$ & $\begin{array}{r}0,1531 \\
0,0416 \\
-0,0130 \\
-0,0001 \\
0,0003 \\
-0,0055\end{array}$ & $\begin{array}{l}0,0019 \\
0,0019 \\
0,0019 \\
0,0019 \\
0,0019 \\
0,0019\end{array}$ & $\begin{array}{r}79,3067 \\
21,5697 \\
-6,7533 \\
-0,0647 \\
0,1547 \\
-2,8535\end{array}$ & $\begin{array}{l}0,0000 \\
0,0000 \\
0,0000 \\
0,9489 \\
0,8782 \\
0,0084\end{array}$ \\
\hline $\begin{array}{l}\text { quadO }_{2} \\
\text { linT.linO } \\
2\end{array}$ & $d d l$ & SCE & $F$ & $P$ \\
\hline $\begin{array}{l}\text { Défaut d'ajustement } \\
\text { Erreur résiduelle }\end{array}$ & $\begin{array}{l}10 \\
16\end{array}$ & $\begin{array}{l}0,05 \cdot 10^{-2} \\
0,26 \cdot 10^{-2}\end{array}$ & 0,30 & 0,96 \\
\hline \multicolumn{3}{|c|}{ Coefficient de détermination } & $R^{2}=0,95$ & \\
\hline
\end{tabular}
Cependant les types d'effet des 2 variables explicatives diffèrent selon les cas.

Tableau I. Modélisation de la vitesse maximale d'acidification $\left(\mathrm{V}_{\mathrm{pH}}\right)$. Maximum acidification rate $\left(V_{p H}\right)$ modelling.

Estimation des coefficients (coef) du modèle reparamétrisé en fonction des polynômes orthogonaux, de leur écart type (std.err), de la valeur du Student (t.stat) et de la probabilité associée ( $\mathrm{P}(\mathrm{t})$ ). L'erreur utilisée pour déterminer l'écart type (std.err) est obtenue en combinant les SCE associées au défaut d'ajustement (10 ddl) et à l'erreur résiduelle (16 ddl). * Un effet est significatif à $95 \%$ si la valeur de la probabilité $\mathrm{P}(\mathrm{t})$ est inférieure à 0,05 . Test de Fisher (F) et probabilité associée $\mathrm{P}$ pour déterminer le manque d'ajustement du modèle. Coefficient de détermination $\mathrm{R}^{2}$.

Estimated coefficients (coef), standard error (std.err), Student value ( $t$.tat) and associated probability $P(t)$ for the model expressed in function of the orthogonal polynomials. The standard error was determined by combining SSE of the lack of fit (10 df) and of the residual emor (16 df). * An effect is significant at a 95\% level if the $P(t)$ value is lower than 0.05 . Fisher test (F) and associated probability $P$ to determine the lack of fit of the model. Determination coefficient $R^{2}$. 
Tableau II. Modélisation de la concentration en diacétyle $\left(C_{D}\right)$.

Diacetyl concentration $\left(C_{D}\right)$ modelling.

\begin{tabular}{|c|c|c|c|c|}
\hline$C_{D}$ & coef & std.err & t.stat & $P(t)$ \\
\hline $\begin{array}{l}\text { Moy* } \\
\text { linT** }^{*} \\
\text { linO }_{2}^{*} \\
\text { quadT }^{*} \\
\text { quadO }_{2}^{*} \\
\text { linT.linO }_{2}{ }^{*}\end{array}$ & $\begin{array}{r}0,0981 \\
-0,0214 \\
0,0878 \\
-0,0014 \\
0,0152 \\
-0,0168\end{array}$ & $\begin{array}{l}0,0015 \\
0,0015 \\
0,0015 \\
0,0015 \\
0,0015 \\
0,0015\end{array}$ & $\begin{array}{r}63,4430 \\
-13,8564 \\
56,7751 \\
-0,9095 \\
9,8239 \\
-10,8836\end{array}$ & $\begin{array}{l}0,0000 \\
0,0000 \\
0,0000 \\
0,3714 \\
0,0000 \\
0,0000\end{array}$ \\
\hline $\begin{array}{l}\text { quadO }{ }_{2}^{*} \\
\text { linT.linO }{ }_{2}^{*}\end{array}$ & $d d l$ & SCE & $F$ & $P$ \\
\hline $\begin{array}{l}\text { Défaut d'ajustement } \\
\text { Erreur résiduelle }\end{array}$ & $\begin{array}{l}10 \\
16\end{array}$ & $\begin{array}{l}0,05 \cdot 10^{-2} \\
0,15 \cdot 10^{-2}\end{array}$ & 0,53 & 0,84 \\
\hline Coefficient de déterm & & $R^{2}=0,99$ & & \\
\hline
\end{tabular}

Estimation des coefficients (coef) du modèle reparamétrisé en fonction des polynômes orthogonaux, de leur écart type (std.err), de la valeur du Student (t.stat) et de la probabilité associée $(P(t))$. L'erreur utilisée pour déterminer l'écart type (std.err) est obtenue en combinant les SCE associées au défaut d'ajustement ( $10 \mathrm{ddl}$ ) et à l'erreur résiduelle (16 ddl). * Un effet est significatif à $95 \%$ si la valeur de la probabilité $P(t)$ est inférieure à 0,05 . Test de Fisher $(F)$ et probabilité associée $P$ pour déterminer le manque d’ajustement du modèle. Coefficient de détermination $\mathrm{R}^{2}$.

Estimated coefficients (coef), standard error (std.err), Student value ( $t$ stat) and associated probability $P(t)$ for the model expressed in function of the orthogonal polynomials. The standard error was determined by combining SSE of the lack of fit (10 df) and of the residual error (16 df). "An effect is significant at a $95 \%$ level if the $P(t)$ value is lower than 0.05 . Fisher test $(F)$ and associated probability $P$ to determine the lack of fit of the model. Determination coefficient $R^{2}$.

Pour $\mathrm{V}_{\mathrm{pH}}$, les effets sont de type linéaire et d'interaction. L'effet linéaire de la température est positif alors que celui de l'oxygène est négatif, mais trois fois moins marqué. Un résultat opposé est observé pour les trois autres variables $\left(C_{D}, C_{A}\right.$ et $\left.D / A\right)$. Les effets quadratiques sont non significatifs au seuil de $95 \%$.

Pour $C_{D}$ et D/A, il n'y a pas d'effet quadratique de la température et l'effet linéaire positif de l'oxygène est 4 fois plus important que celui négatif de la température. Remarquons aussi que l'effet d'interaction est presque aussi prononcé que l'effet linéaire de la température. Cela traduit le fait que l'influence de la température est d'autant plus marquée que la concentration en oxygène est élevée.

Pour $C_{A}$, la température n'a pas d'effet linéaire et l'effet linéaire de l'oxygène est 5 fois plus important que les autres effets. $C_{A}$ est aussi la seule variable pour laquelle l'effet d'interaction de la température et de l'oxygène se révèle positif. Cela signifie que pour cet effet, il y a augmentation de la production d'acétoïne lorsque la température et l'oxygène varient dans le même sens, alors que pour les autres variables il faut faire varier les deux facteurs dans des sens opposés. 
Tableau III. Modélisation de la concentration en acétoïne $\left(\mathrm{C}_{A}\right)$. Acetoin concentration $\left(C_{A}\right)$ modelling.

\begin{tabular}{|c|c|c|c|c|}
\hline$C_{A}$ & coef & std.err & t.stat & $P(t)$ \\
\hline $\begin{array}{l}\text { Moy * } \\
\text { linT } \\
\operatorname{linO}_{2}{ }^{*} \\
\text { quadT * } \\
\text { quadO } \\
\text { linT.linO }_{2}{ }^{*}\end{array}$ & $\begin{array}{r}4,2553 \\
-0,0675 \\
0,7474 \\
-0,1597 \\
-0,1500 \\
0,1076\end{array}$ & $\begin{array}{l}0,0458 \\
0,0458 \\
0,0458 \\
0,0458 \\
0,0458 \\
0,0458\end{array}$ & $\begin{array}{r}92,8661 \\
-1,4731 \\
16,3101 \\
-3,4850 \\
-3,2740 \\
2,3482\end{array}$ & $\begin{array}{l}0,0000 \\
0,1527 \\
0,0000 \\
0,0018 \\
0,0030 \\
0,0267\end{array}$ \\
\hline $\operatorname{linT} \cdot \operatorname{lin} \mathrm{O}_{2}{ }^{*}$ & $d d l$ & SCE & $F$ & $P$ \\
\hline $\begin{array}{l}\text { Défaut d'ajustement } \\
\text { Erreur résiduelle }\end{array}$ & $\begin{array}{l}10 \\
16\end{array}$ & $\begin{array}{l}0,87 \\
0,87\end{array}$ & 0,62 & 0,81 \\
\hline \multicolumn{3}{|c|}{ Coefficient de détermination } & $R^{2}=0,92$ & \\
\hline
\end{tabular}

Estimation des coefficients (coef) du modèle reparamétrisé en fonction des polynômes orthogonaux, de leur écart type (std.err), de la valeur du Student (t.stat) et de la probabilité associée $(P(t))$. L'erreur utilisée pour déterminer l'écart type (std.err) est obtenue en combinant les SCE associées au défaut d'ajustement ( $10 \mathrm{ddl}$ ) et à l'erreur résiduelle (16 ddl). * Un effet est significatif à $95 \%$ si la valeur de la probabilité $P(t)$ est inférieure à 0,05 . Test de Fisher (F) et probabilité associée $\mathrm{P}$ pour déterminer le manque d'ajustement du modèle. Coefficient de détermination $\mathrm{R}^{2}$.

Estimated coefficients (coef), standard error (std.err), Student value (t.stat) and associated probability P(t) for the model expressed in function of the orthogonal polynomials. The standard error was determined by combining SSE of the lack of fit (10 df) and of the residual error (16 df). "An effect is significant at a 95\% level if the $P(t)$ value is lower than 0.05 . Fisher test (F) and associated probability $P$ to determine the lack of fit of the model. Determination coefficient $R^{2}$.

\section{Représentation graphique des surfaces de réponse}

Les figures $1 A$ à $D$ représentent les surfaces de réponse relatives respectivement à $V_{p H}, C_{D}, C_{A}$ et D/A.

La figure $1 \mathrm{~A}$ confirme l'effet prépondérant de la température sur la vitesse maximale d'acidification par rapport à l'effet de la concentration initiale en oxygène. La vitesse d'acidification maximum $(0,23$ unité $\mathrm{pH} / \mathrm{h})$ est située à une des extrémités du plan d'expérience. Dans le cas présent, elle correspond à $0 \%$ d'oxygène et à une température de $30^{\circ} \mathrm{C}$.

D'après la figure $1 \mathrm{~B}$, la production maximale en diacétyle est beaucoup plus sen- sible aux changements de la concentration initiale en oxygène qu'à ceux de la température. La surface présente une courbure dirigée vers le haut suivant l'axe oxygène. La concentration maximale en diacétyle $(0,31 \mathrm{mmol} / \mathrm{l})$ est obtenue sur une valeur extrême du plan d'expérience, pour $100 \%$ d'oxygène et à une température de $18^{\circ} \mathrm{C}$.

La figure $1 \mathrm{C}$ montre que la production maximale d'acétoïne est également plus sensible aux variations d'oxygène que de température. La surface présente une courbure dirigée vers le bas selon les 2 axes. Cette surface présente alors un maximum global pour une concentration théorique en oxygène située en dehors du plan d'expérience et à une température de $26^{\circ} \mathrm{C}$. La 
Tableau IV. Modélisation du rapport diacétyle/acétoïne (D/A).

Ratio diacetyl/acetoin (D/A) modelling.

\begin{tabular}{|c|c|c|c|c|}
\hline$D / A$ & coef & std.err & t.stat & $P(t)$ \\
\hline $\begin{array}{l}\text { Moy * } \\
\text { linT* } \\
\operatorname{linO}_{2}{ }^{*} \\
\text { quadT } \\
\text { quadO }{ }_{2}^{*} \\
\text { linT.linO }_{2}\end{array}$ & $\begin{array}{r}2,0394 \\
-0,4511 \\
1,6001 \\
0,0106 \\
0,2135 \\
-0,3270\end{array}$ & $\begin{array}{l}0,0279 \\
0,0279 \\
0,0279 \\
0,0279 \\
0,0279 \\
0,0279\end{array}$ & $\begin{array}{r}73,1092 \\
-16,1724 \\
57,3612 \\
0,3809 \\
7,6533 \\
-11,7212\end{array}$ & $\begin{array}{l}0,0000 \\
0,0000 \\
0,0000 \\
0,7064 \\
0,0000 \\
0,0000\end{array}$ \\
\hline $\operatorname{linT} \cdot \operatorname{lin} \mathrm{O}_{2}{ }^{*}$ & $d d l$ & SCE & $F$ & $P$ \\
\hline $\begin{array}{l}\text { Défaut } \\
\text { d'ajustement } \\
\text { Erreur résiduelle }\end{array}$ & $\begin{array}{l}10 \\
16\end{array}$ & $\begin{array}{l}0,25 \cdot 10^{-2} \\
0,39 \cdot 10^{-2}\end{array}$ & 0,99 & 0,49 \\
\hline \multicolumn{3}{|c|}{ Coefficient de détermination } & $R^{2}=0,99$ & \\
\hline
\end{tabular}

Estimation des coefficients (coef) du modèle reparamétrisé en fonction des polynômes orthogonaux, de leur écart type (std.err), de la valeur du student (t.stat) et de la probabilité associée $(P(t))$. L'erreur utilisée pour déterminer l'écart type (std.err) est obtenue en combinant les SCE associées au défaut d'ajustement (10 ddl) et à l'erreur résiduelle (16 ddl). - Un effet est significatif à $95 \%$ si la valeur de la probabilité $P(t)$ est iniérieure à 0,05 . Test de Fisher $(F)$ et probabilité associée $\mathrm{P}$ pour déterminer le manque d'ajustement du modèle. Coefficient de détermination $\mathrm{R}^{2}$.

Estimated coefficients (coef), standard error (std.err), Student value ( $t$ stat) and associated probability $P(t)$ for the model expressed in function of the orthogonal polynomials. The standard error was determined by combining SSE of the lack of fit (10 df) and of the residual error (16 df). "An effect is significant at a $95 \%$ level if the $P(t)$ value is lower than 0.05 . Fisher test (F) and associated probability $P$ to determine the lack of fit of the model. Determination coefficient $R^{2}$.

concentration maximale en acétoïne $(5,3$ $\mathrm{mmol} / \mathrm{l}$ ) est donc obtenue à $26^{\circ} \mathrm{C}$ et $100 \%$ d'oxygène (figure $1 \mathrm{C}$ ).

La figure $1 \mathrm{D}$ montre que le rapport $\mathrm{D} / \mathrm{A}$ est lui aussi plus sensible à l'oxygène qu'à la température. Le rapport D/A maximum $(5,94 \%)$ est obtenu à $100 \%$ d'oxygène et à une température de $18^{\circ} \mathrm{C}$.

\section{DISCUSSION}

L'analyse des effets linéaires, quadratiques et d'interaction de la température et de l'oxygène sur les variables expliquées $\left(V_{p H}, C_{D}\right.$, $\mathrm{C}_{\mathrm{A}}$ et $\mathrm{D} / \mathrm{A}$ ) a montré que l'interaction de ces
2 grandeurs est observée de façon systématique. Ce résultat démontre que la température et la concentration en oxygène ont des effets qui ne s'additionnent pas mais qui s'amplifient mutuellement sur la production de diacétyle ou d'acétoïne et sur la vitesse d'acidification. Ce phénomène d'interaction peut avoir une cause physicochimique car la solubilité de l'oxygène augmente lorsque la température diminue. Ainsi, Atkinston et Mavituna (1983) ont montré que la solubilité de l'oxygène dans l'eau à $15^{\circ} \mathrm{C}(1,54 \mathrm{mmol} / \mathrm{l})$ est 1,3 fois plus élevée qu'à $30^{\circ} \mathrm{C}(1,16 \mathrm{mmol} / \mathrm{l})$. Cela expliquerait que, par exemple, l'effet positif de l'oxygène sur la production de diacétyle soit encore plus marqué à $18^{\circ} \mathrm{C}$ qu'à $30^{\circ} \mathrm{C}$ (fig $1 \mathrm{~B}$ ). 

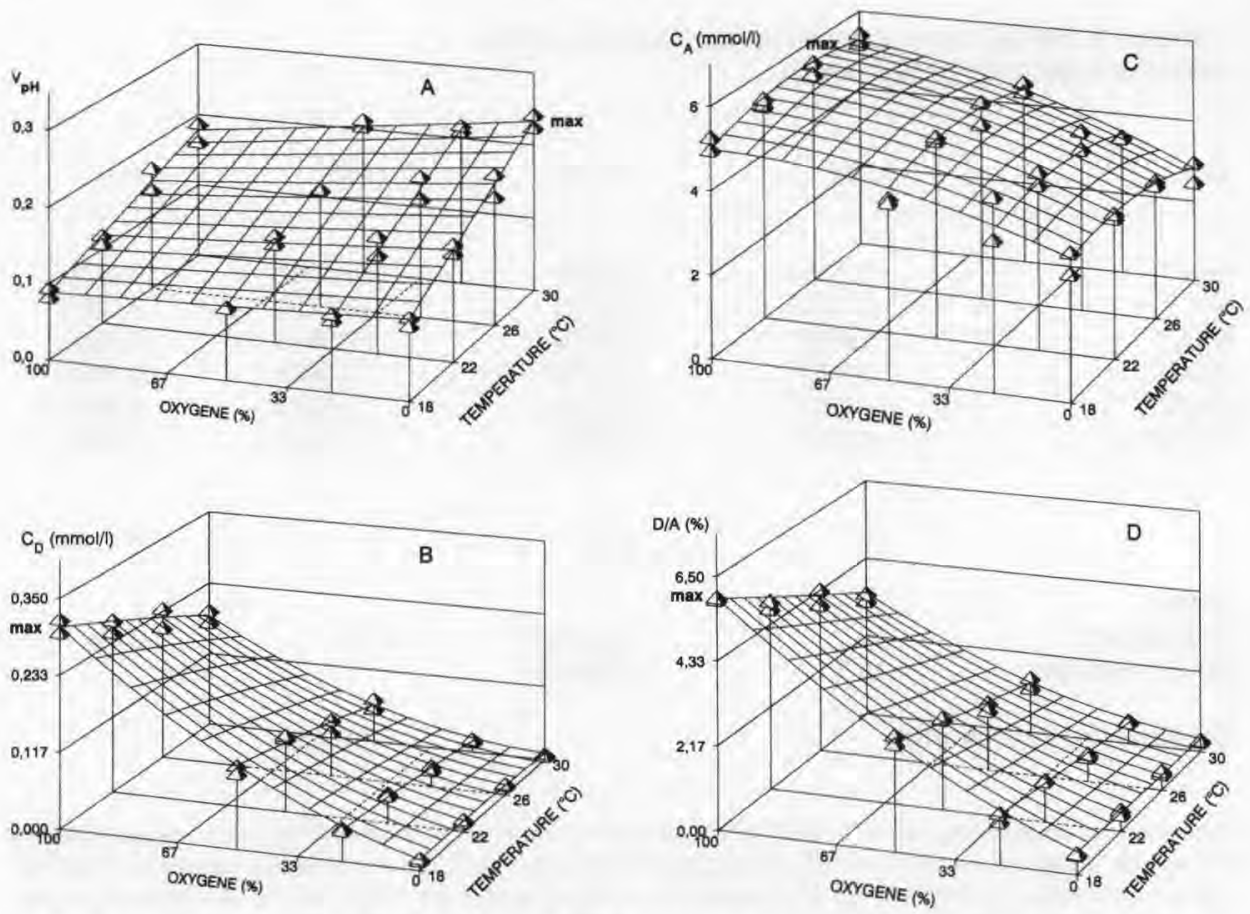

Fig 1. Surfaces de réponse traduisant l'effet de la concentration initiale en oxygène (exprimée en pourcentage de la saturation du milieu par l'oxygène) et de la température sur la vitesse maximale d'acidification (A), la concentration maximale en diacétyle (B), la concentration maximale en acétoïne (C) et le rapport $D / A(D)$ (max : maximum de la surface de réponse ; les symboles en forme de pyramide indiquent les points expérimentaux).

Response surface showing the effects of the initial oxygen concentration (expressed in percentage of the medium saturation by oxygen) and of the temperature on maximum acidification rate (A), maximum diacetyl concentration (B), maximum acetoin concentration (C) and diacetyl/acetoin ratio D/A (D). (max: maximum of the response surface; the experimental points are represented by pyramidal symbols).

Quant à $\mathrm{V}_{\mathrm{pH}}$, l'effet inhibiteur de l'oxygène sur l'acidification est visible à $30^{\circ} \mathrm{C}$ (fig $1 \mathrm{~A}$ ). En revanche, il est peu marqué à $18^{\circ} \mathrm{C}$ alors que la solubilité de l'oxygène est plus importante. De la même façon, l'effet positif de l'oxygène sur la production d'acétoïne, observé à $30^{\circ} \mathrm{C}$, n'est pas renforcé à $18^{\circ} \mathrm{C}$ (fig 1C). Ainsi, l'interaction entre l'oxygène et la température ne s'explique pas uniquement par la solubilité plus importante de ce gaz à des températures basses. II doit s'agir de phénomènes plus complexes d'activation et d'inhibition au niveau de la synthèse ou de l'activité des enzymes régissant le métabolisme des bactéries lactiques.
Le métabolisme du citrate qui conduit au diacétyle et à l'acétoïne est maintenant bien connu (Harvey et Collins, 1962, 1963 ; Verhue et Tjan, 1991). Le pyruvate constitue un des composés clefs (fig 2). II est transformé en acétolactate par une acétolactate synthétase. L'acétolactate est transformé soit en diacétyle, soit en acétoïne par l'acétolactate décarboxylase. Le diacétyle peut être réduit en acétoïne par la diacétyle réductase et ce dernier en 2,3 butanediol par l'acétoïne réductase. L'oxygène intervient donc directement dans la production de diacétyle car la transformation de l'acétolactate en diacétyle est une décarboxyla- 
tion oxydative (Stadhouders, 1974 ; Hugenholtz et Starrenburg, 1992). Il a été montré que l'oxygène favorisait la synthèse de l'acétolactate synthétase et de la NADH oxydase (Bassit et al, 1993). Cette dernière enzyme assurerait pour partie le rôle de la lactate déshydrogénase, de la diacétyle réductase et de l'acétoïne réductase dans la réoxydation du NADH, permettant ainsi l'accumulation de l'acétoïne et du diacétyle. De plus, l'augmentation de la température favorise la synthèse de la diacétyle réductase et inhibe celle de la NADH oxydase (Petit et al, 1989 ; Bassit et al, 1994). Tout cela montre que la température et l'oxygène peuvent avoir des effets complexes, qui s'amplifient ou s'opposent, sur la production de diacétyle et d'acétoïne. L'ensemble de ces effets est représenté sur la figure 2. Les résultats obtenus avec $L$ lactis subsp lactis biovar diacetylactis CNRZ 483 mon-

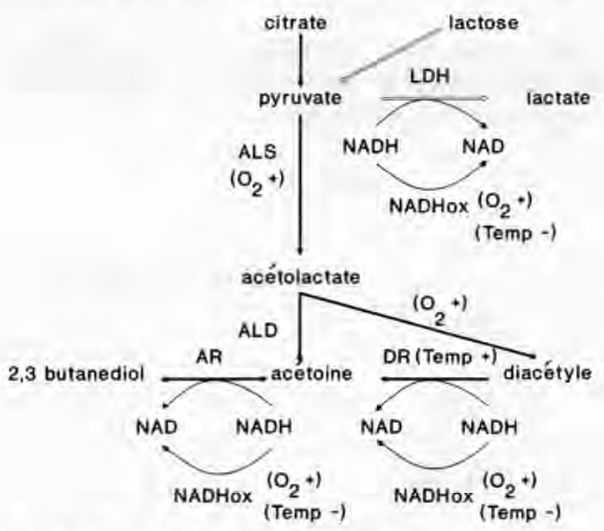

Fig 2. Effet positif $(+)$ ou négatif ( - ) de la température (Temp) et de l'oxygène $\left(\mathrm{O}_{2}\right)$ sur la synthèse des principales enzymes du métabolisme du citrate (ALS : acétolactate synthétase ; ALD : acétolactate décarboxylase ; $\mathrm{LDH}$ : lactate déshydrogénase ; NADHox : NADH oxydase : DR : diacétyle réductase; $A R$ : acétoïne réductase). Positive $(+)$ or negative $(-)$ effect of temperature (Temp) and oxygen $\left(\mathrm{O}_{2}\right)$ on the synthesis of the main enzymes involved in citrate metabolism (ALS: acetolactate synthetase; ALD: acetolactate decarboxylase; $L D H$; lactate dehydrogenase; NADHox: NADH oxidase; DR: diacetyl reductase; $A R$ : acetoin reductase). trent que l'effet de la température sur la concentration maximale en acétoïne est faible. Ces résultats sont en accord avec ceux d'Oberman et al (1982) qui n'ont trouvé aucune différence de production d'acétoine pour des cultures réalisées à des températures de $23^{\circ} \mathrm{C}$ et de $28^{\circ} \mathrm{C}$. De même, Schmitt et al (1988) ont conclu que la température n'a pas d'effet sur le niveau d'acétoïne, qui reste relativement constant pour les 2 températures testées ( 22 et $34^{\circ} \mathrm{C}$ ).

D'une manière générale, les résultats observés avec Lactococcus lactis subsp lactis biovar diacetylactis CNRZ 483, semblent s'appliquer à d'autres souches de lactocoques. Seule l'ampleur des phénomènes observés peut varier d'une souche à une autre (Lee et Collins, 1975 ; Petit et al, 1989; Bassit et al, 1993). En revanche, it est impossible d'affirmer que l'ensemble des résultats seraient encore valides en culture mixte où des souches acidifiantes (L lactis subsp lactis ou subsp cremoris) sont associées à des souches aromatisantes (L lactis subsp lactis biovar diacetylactis). En effet, il existe alors des interactions bactériennes susceptibles de modifier notamment la production de diacétyle et d'acétoïne. La présence de diacétyle et d'acétoïne réductases actives chez les sous-espèces lactis et cremoris et donc capables de réduire le diacétyle et l'acétoïne, est un exemple d'interaction possible parmi d'autres.

En résumé, l'effet des 2 facteurs étudiés (la concentration initiale en oxygène et la température de culture) montre que la vitesse maximale d'acidification la plus importante est observée à $30^{\circ} \mathrm{C}$ et en absence d'oxygène ; et que la concentration maximale en diacétyle et le rapport D/A sont maximums à une température de $18^{\circ} \mathrm{C}$ et en présence de $100 \%$ en oxygène.

II existe donc un antagonisme entre les conditions de culture favorables à l'acidification du milieu d'une part, et à la production de diacétyle d'autre part. Aussi, en fonction des objectifs recherchés (acidification rapide 
du caillé et/ou obtention d'un caillé riche en diacétyle) il est possible de définir des conditions optimales de conduite du procédé de production de fromages frais.

\section{REMERCIEMENTS}

Les auteurs remercient $\mathrm{G}$ Yonnet et $\mathrm{E}$ Ferreira pour leur excellente assistance technique.

\section{ANNEXE - Détermination des poly- nômes orthonormés}

\section{Calcul des polynômes orthonormés du facteur $T$}

Dans le cas d'une répartition équidistante des essais, la détermination des polynômes orthonormés est très simplifiée si on effectue un codage du facteur pour obtenir des valeurs symétriques autour de 0 d'après l'équation suivante :

$$
T_{C}=\frac{(T-24)}{2}
$$

Les valeurs codées de $T_{C}$ prennent alors les valeurs $-3,-1,1$ et 3 correspondant à $18,22,26$ et $30^{\circ} \mathrm{C}$. Soit $P_{0}, P_{1}$ et $P_{2}$ les polynômes orthonormés relatifs à $T_{c}$, on a alors :

$$
\begin{gathered}
P_{0}\left(T_{c}\right)=1 \\
P_{1}\left(T_{c}\right)=\frac{T_{c}}{\sqrt{m_{2}}} \\
P_{2}\left(T_{c}\right)=\frac{\left(T_{c}^{2}-m_{2}\right)}{\sqrt{m_{4}-m_{2}^{2}}}
\end{gathered}
$$

avec

$$
\begin{aligned}
& m_{2}=\frac{\left((-3)^{2}+(-1)^{2}+1^{2}+3^{2}\right)}{4}=\frac{20}{4}=5 \\
& m_{4}=\frac{\left((-3)^{4}+(-1)^{4}+1^{4}+3^{4}\right.}{4}=\frac{164}{4}=41
\end{aligned}
$$

d'où :

$$
\begin{aligned}
& P_{1}\left(T_{c}\right)=\frac{T_{c}}{\sqrt{5}} \\
& P_{2}\left(T_{c}\right)=\frac{T_{c}^{2}}{4}-\frac{5}{4}
\end{aligned}
$$

En remplaçant $T_{c}$ par $T$, on obtient :

$$
\begin{aligned}
& P_{1}(T)=\frac{T-24}{2 \sqrt{5}} \\
& P_{2}(T)=\frac{T^{2}}{16}-3 T+\frac{139}{4}
\end{aligned}
$$

\section{Calcul des polynômes orthonormés du facteur $\mathrm{O}_{2}$}

Dans le cas d'une répartition non équidistante des essais la détermination des polynômes orthonormés est plus complexe.

$$
Q_{0}\left(O_{2}\right)=1
$$

$$
Q_{1}=\frac{Q_{1}{ }^{\prime}}{\sqrt{\left\langle Q_{1}{ }^{\prime}, Q_{1}{ }^{\prime}\right\rangle}} \quad Q_{1}{ }^{\prime}\left(O_{2}\right)=O_{2}-\left\langle O_{2}, 1\right\rangle
$$

$Q_{2}=\frac{Q_{2}^{\prime}}{\sqrt{\left\langle Q_{2}^{\prime}, Q_{2}^{\prime}\right\rangle}}$

$$
Q_{2}^{\prime}\left(O_{2}\right)=O_{2}^{2}-\left\langle O_{2}^{2}, 1\right\rangle-\frac{\left\langle O_{2}^{2}, Q_{1}{ }^{\prime}\right\rangle}{\left\langle Q_{1}^{\prime}, Q_{1}^{\prime}\right\rangle} \cdot Q_{1}^{\prime}
$$

La notation $\langle A, B\rangle$ indique qu'il s'agit du produit scalaire entre les 2 polynômes $A$ et $B, \sqrt{ }\langle A, B\rangle$ étant la norme associée à ce produit scalaire.

En posant $m_{i}=1 / 4\left(0^{i}+21^{i}+50^{i}+100^{i}\right)$, il est possible de réécrire les polynômes $Q_{i}$ en fonction des coefficients $m_{i}$ :

$$
Q_{1}\left(O_{2}\right)=\frac{O_{2}-m_{1}}{\sqrt{m_{2}-m_{1}^{2}}}
$$


$Q_{2}\left(O_{2}\right)=\frac{O_{2}^{2}-\frac{\left(m_{3}-m_{2} m_{1}\right)}{\left(m_{2}-m_{1}^{2}\right)} O_{2}+\frac{\left(m_{1} m_{3}-m_{2}^{2}\right)}{\left(m_{2}-m_{1}^{2}\right)}}{\sqrt{m_{4}+\frac{\left(2 m_{1} m_{2} m_{3}-m_{3}^{2}-m_{2}^{3}\right)}{\left(m_{2}-m_{1}^{2}\right)}}}$

\section{Calcul des polynômes orthonormés des 2 facteurs $\mathrm{T}$ et $\mathrm{O}_{2}$}

Les polynômes $R_{i j}$ orthonormés de $T$ et $\mathrm{O}_{2}$ sont le résultat du produit tensoriel des polynômes $\mathrm{P}_{\mathrm{i}}$ et $Q_{\mathrm{j}}$. Ainsi, $R_{i j}\left(T, O_{2}\right)=P_{i}(T) \otimes Q_{j}\left(O_{2}\right)$

d'où :

$$
Y\left(T, O_{2}\right)=\alpha_{00}+\alpha_{10} P_{1}(T)+\alpha_{01} Q_{1}\left(O_{2}\right)
$$$$
+\alpha_{20} P_{2}(T)+\alpha_{02} Q_{2}\left(O_{2}\right)+\alpha_{11} P_{1}(T) Q_{1}\left(O_{2}\right)
$$

Après remplacement des polynômes $P_{\mathrm{i}}$ et $Q_{\mathrm{j}}$, on obtient :

$$
\begin{gathered}
Y\left(T, O_{2}\right)=\alpha_{00}+\alpha_{10}(0,224 \cdot T-5,366) \\
+\alpha_{01}\left(0,0266 \cdot O_{2}-1,1394\right)+\alpha_{20}(0,0625 \\
\left.\cdot T^{2}-3 \cdot T+34,75\right)+\alpha_{02}\left(0,00093 \cdot O_{2}^{2}\right. \\
\left.-0,096 \cdot O_{2}+1,0943\right)+\alpha_{11}(0,224 \cdot T-5,366) \\
\left(0,0266 \cdot O_{2}-1,1394\right)+\varepsilon
\end{gathered}
$$

\section{RÉFÉRENCES}

Atkinston B, Mavituna F (1983) Biochemical Engineering, Biotechnology Handbook. Macmillan Publishers Ltd. The nature press, New York

Bassit N, Boquien CY, Picque D, Corrieu G (1993) Effect of initial oxygen concentration on diacetyl and acetoin production by Lactococcus lactis subsp lactis biovar diacetylactis. Appl Environ Microbiol 59, 1893-1897

Bassit N, Boquien CY, Picque D, Corrieu G (1994) Effect of temperature on diacetyl and acetoin production by Lactococcus lactis subsp lactis biovar diacetylactis CNRZ 483 (Submitted to J Dairy Res)

Harvey RJ, Collins EB (1962) Citrate transport system of Streptococcus diacetylactis. J Bacteriol 83, 1005-1009

Harvey RJ, Collins EB (1963) Roles of citrate and acetoin in the metabolism of Streptococcus diacetylactis. J Bacteriol 86, 1301-1307
Hugenholtz J, Starrenburg MJC (1992) Diacetyl production by different strains of Lactococcus lactis subsp lactis var diacetylactis and Leuconostoc spp. Appl Microbiol Biotechnol 38, 17-22

Kaneko T, Takahashi M, Suzuki H (1990) Acetoin fermentation by citrate-positive Lactococcus lactis subsp lactis 3022 grown aerobically in the presence of hemin or $\mathrm{Cu}^{2+}$. Appl Environ Microbiol 56, 2644-2649

Kobilinsky A (1988) Tactiques en analyse de variance et en régression. Rev Modulad 1 , 25-58

Lebreton JD, Millier C (1982) Courbes de réponse croissantes avec point d'inflexion. In: Modèles dynamiques déterministes en biologie. Masson, Paris, 162-164

Lee DA, Collins EB (1975) Influences of temperature on growth of Streptococcus cremoris and Streptococcus lactis. J Dairy Sci59, 405409

Noël Y, Durier C, Lehembre N, Kobilinsky A (1991) Étude multifactorielle de la coagulation mixte du lait analysée par viscoélasticimétrie. Lait 71, 15-39

Oberman H, Piatkiewicz A, Libudzisz Z (1982) Production of diacetyl and acetoin by lactic acid bacteria. Nahrung 26, 615-623

Ochi H, Takahashi M, Kaneko T, Suzuki H, Tanaka H (1991) Diacetyl production by coimmobilized citrate-positive Lactococcus lactis subsp lactis 3022 and homogenized bovine liver in alginate fibers with double gel layers. Biotechnol Lett 13, 505-510

Petit C, Vilchez F, Marczak R (1989) Formation and stabilization of diacetyl and acetoin concentration in fully grown cultures of Streptococcus lactis subsp diacetylactis. Biotechnol Lett 11, 53-56

Schmitt $P$, Couvreur $C$, Cavin JF, Prévost $H$, Divies C (1988) Citrate utilisation by free and immobilized Streptococcus lactis subsp diacetylactis in continuous culture. Appl Microbiol Biotechnol 29, 430-436

Spinnler HE, Corrieu G (1989) Automatic method to quantify starter activity based on $\mathrm{pH}$ measurement. J Dairy Res 56, 755-764

Stadhouders J (1974) Dairy starter cultures. Milchwissenschaft 29, 329-337

Takahashi $H$, Ochi $H$, Kaneko $T$, Suzuki $H$, Tanaka H (1990) Diacetyl production by immobilized citrate-positive Lactococcus lactis subsp 
lactis 3022 in fibrous Ca-alginate gel. Biotechnol Lett 12, 569-574

Verhue WM, Tjan FSB (1991) Study of the citrate metabolism of Lactococcus lactis subsp lactis biovar diacetylactis by means of ${ }^{13} \mathrm{C}$ nuclear magnetic resonance. Appl Environ Microbiol $57,3371-3377$
Walsh B, Cogan TM (1974) Separation of diacetyl and acetoin in milk. J Dairy Res 41, 25-30

Xanthopoulos V, Picque D, Bassit N, Boquien CY, Corrieu G (1994) Methods for determination of aroma compounds in dairy products: a comparative study. J Dairy Res (accepté pour publication) 